\title{
Development of Th1 Imprints to rBCG Expressing a Foreign Protein: Implications for Vaccination against HIV-1 and Diverse Influenza Strains
}

\author{
Carl Power, ${ }^{1,2}$ Travis W. Marfleet, ${ }^{2}$ Louis Qualtiere, ${ }^{2,3}$ Wei Xiao, ${ }^{2}$ and Peter Bretscher ${ }^{2}$ \\ ${ }^{1}$ Prince of Wales Clinical School, University of New South Wales, NSW2052 Sydney, Australia \\ ${ }^{2}$ Department of Microbiology and Immunology, University of Saskatchewan, Saskatoon, SK, Canada S7N5E5 \\ ${ }^{3}$ Department of Pathology and Laboratory Medicine, University of Saskatchewan, Saskatoon, SK, Canada S7NOW8
}

Correspondence should be addressed to Peter Bretscher, peter.bretscher@usask.ca

Received 5 December 2009; Revised 14 March 2010; Accepted 22 March 2010

Academic Editor: Hanchun Yang

Copyright () 2010 Carl Power et al. This is an open access article distributed under the Creative Commons Attribution License, which permits unrestricted use, distribution, and reproduction in any medium, provided the original work is properly cited.

\begin{abstract}
We demonstrate here that immunizing naïve mice with low numbers of recombinant Bacille Calmette-Guérin (rBCG) expressing $\beta$-galactosidase $(\beta$-gal) generates predominant Th1 responses to both BCG and $\beta$-gal whereas infection with high numbers generates a mixed Th1/Th2 response to both BCG and $\beta$-gal. Furthermore, the Th1 response to both BCG and $\beta$-gal is stable when mice, pre-exposed to low numbers of rBCG, are challenged four months later with high numbers of rBCG. Thus the Th1/Th2 phenotypes of the immune responses to $\beta$-gal and to BCG are "coherently" regulated. Such rBCG vectors, encoding antigens of pathogens preferentially susceptible to cell-mediated attack, may be useful in vaccinating against such pathogens. We discuss vaccination strategies employing $\mathrm{rBCG}$ vectors that are designed to provide protection against diverse influenza strains or numerous variants of HIV-1 and consider what further experiments are essential to explore the possibility of realizing such strategies.
\end{abstract}

\section{Introduction}

A rational approach to vaccination against a pathogen is contingent upon a knowledge of the immunological correlates that discriminate between immunity able to contain the pathogen and immunity that fails to do so. Effective vaccination must guarantee the former type of immunity under circumstance where the latter type of immunity would be generated upon natural infection of the naïve individual [1]. Effective vaccination of people, against a diversity of pathogens, is achieved through guaranteeing a rapid and large antibody, Th2 response upon natural infection by the pathogen. Such vaccination is effective only when antibody is able to contain the pathogen and/or neutralize the toxins the pathogen produces. However, there are other situations, following infection by some intracellular pathogens or the development of cancer, where the correlates of protection are a predominant cell-mediated, CTL, Th1 response and where responses with a substantial Th2, antibody component, are associated with chronic or progressive disease [2]. In these cases, it would appear that effective vaccination must generate a Th1 imprint upon the immune system that is stable upon natural infection by the pathogen [3].

Mycobacterium bovis is responsible for tuberculosis of cattle. An attenuated form of $M$. bovis, named BCG, is currently the only available vaccine against Mycobacterium tuberculosis, the pathogen primarily responsible for human tuberculosis. Billions of individuals worldwide have been inoculated with BCG in attempts to provide protection against tuberculosis and leprosy. Moreover, these bacteria can be used as vectors that express antigens of diverse pathogens to raise immunity against these antigens. Several recombinant BCG vectors have been constructed for vaccination against a variety of bacterial, viral, or parasitic pathogens [4].

There are several advantages in using rBCG vectors. Not only does BCG act as an adjuvant, but its slow replication allows for persistent, low level delivery of antigen to the 
host over a considerable time. Many observations show, in diverse systems, that chronic stimulation with low amounts of antigen can lead to the establishment of Th1 imprints $[2,3,5-7]$. Additionally, advances in expression and delivery of antigen by $\mathrm{rBCG}$, hence increasing the availability of the encoded antigens to the host immune system, have allowed the development of vaccine vectors superior to those of the past [4]. We are also encouraged to explore the use of rBCG vectors in developing strategies of vaccination by the remarkable safety record of BCG as a vaccination agent [8].

A critical question, when considering the use of $\mathrm{rBCG}$ vectors to immunize against the encoded protein, is the relationship between the nature of the immune response to BCG and the nature of the response to the encoded protein. This is particularly critical in those cases where there are grounds for believing that the Th1/Th2 phenotype of the response to the encoded antigen is likely critical to the degree of protection provided by vaccination. It has been proposed that the Th1/Th2 phenotype of the responses to BCG and the encoded protein may be coordinately determined [1], a phenomenon referred to as coherence. We set out to explore whether, employing $\mathrm{rBCG}$ encoding $\beta$-galactosidase as a model $\mathrm{rBCG}$ vector, the Th1/Th2 phenotypes of the immune responses to BCG and to $\beta$-galactosidase are coherently regulated. Should this be the case, it would lay the foundation for further studies aimed at examining whether $\mathrm{rBCG}$ vectors could be employed to establish Th1 imprints against those pathogens preferentially susceptible to predominant cellmediated, Th1 responses.

\section{Materials and Methods}

2.1. Mice. Female mice were used in all experiments. $\mathrm{BALB} / \mathrm{cJ}$ mice were obtained from the animal colony at the Department of Microbiology and Immunology, University of Saskatchewan. All mice were housed at the animal care facility within the Department and were at least 6 weeks old at the time of immunization. Animal care and treatment were in accordance with standards approved by the Canadian Council on Animal Care.

2.2. Growth and Enumeration of $r B C G$. Dr. Emil Skamene of McGill University provided M. bovis strain BCG Montreal. The mycobacteria were propagated in Dubos medium containing $0.5 \%$ bovine serum albumin and $0.05 \%$ Tween 80. Bacteria were enumerated by plating various dilutions on Dubos agar plates after sonication for five seconds at a power output of 5 and $50 \%$ duty cycle to break up clusters of bacteria. Colonies were counted fourteen days after plating, and consequently numbers of bacteria are expressed as cfu.

2.3. Immunizations. Fourteen-day cultures of BCG or rBCG were used for injections. The cells were pelleted by centrifugation at $8000 \mathrm{~g}$ for 30 minutes and washed three times by repeated resuspension in saline containing $0.05 \%$ Tween 80 followed by centrifugation at $8000 \mathrm{~g}$ for 20 minutes. After the final wash, the mycobacteria were resuspended in $1 / 100$ of the original volume of culture, and serial dilutions were performed from this stock as needed. BCG immunizations were performed by either intradermal (i.d.) or intravenous (i.v.) route. For i.d. injection, hair was removed from the abdomen using a commercial depilatory two days prior to injection. Injections were done using a $1 \mathrm{cc}$ syringe with a 30-gauge needle attached. Mice were anesthetized, and the skin of the abdomen was gently stretched to prevent it from bunching up in front of the needle. With the bevel of the needle facing upward, the needle was inserted several millimeters horizontally into the skin and $25-50 \mu \mathrm{l}$ of the BCG suspension containing the appropriate number of cfu was injected. For i.v. injection, the desired number of BCG cfu were injected in a $50 \mu \mathrm{l}$ volume into the lateral tail vein using a 28 -gauge needle.

Mice were immunized by the i.p. route with recombinant $\beta$-gal (Sigma Chemicals, St. Louis, MO), either as a soluble protein in phosphate-buffered saline (PBS) or adsorbed to the aluminum hydroxide gel adjuvant, Alhydrogel (Superfos Biosector, Vedbaek, Denmark) as indicated in the legend to Figure 6.

2.4. ELISPOT Assay for Detection and Enumeration of Antigen-Dependent IFN $\gamma$ - and IL-4-Secreting Cells. The ELISPOT assay for enumeration of BCG-specific cytokine producing lymphocytes was performed on spleen cells as previously described in detail [9]. For detection of $\beta$-galspecific cytokine-producing cells, the procedure was identical except that purified soluble $\beta$-gal (Sigma Chemicals) was used at $1 \mu \mathrm{g} /$ well for stimulation of lymphocytes.

2.5. Analysis of Antimycobacterial or Anti- $\beta$-gal Antibodies by Enzyme Immunoassay. Blood from normal or infected mice was collected by tail bleeding into serum separator tubes (Becton Dickinson, Rutherford, NJ). After allowing the samples to clot for at least several hours, individual serum samples were harvested by centrifugation at approximately $5000 \mathrm{~g}$ for 60 seconds. Sera were stored at $-70^{\circ} \mathrm{C}$ until use. The IgG1 and IgG2a serum antibody titers were determined using an ELISA. Immulon-4 96-well polystyrene plates (Dynatech Laboratories, Chantilly, VA) were coated with either BCG antigen or with soluble $\beta$-gal antigen at a concentration of $1 \mu \mathrm{g} / \mathrm{well}$ in $100 \mu \mathrm{l}$ of PBS and the procedure was performed as described in [10]. The titer was determined by plotting optical density against the log of the dilution factor for each sample. The endpoint titer was assigned as the point on the line at which the optical density was 0.5 above the value for wells to which no serum was added.

2.6. Mycobacterial Expression Vectors. Dr. Mark Hanson of MedImmune, Gaithersberg, MA, kindly provided the mycobacterial expression vector pMV361::LacZ [11]. Vector pMV361 is a chromosomal integrating vector in which inserted genes are expressed as a fusion protein with the first six amino acids of the mycobacterial heat shock protein 60 (Hsp60).

2.7. Transformation of Competent M. bovis BCG. The method of Parish and Stoker [12] was used to prepare 


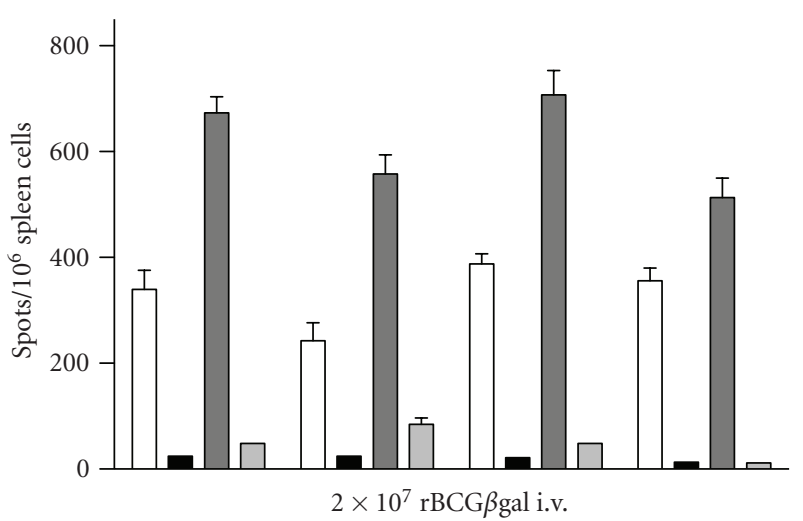

(a)

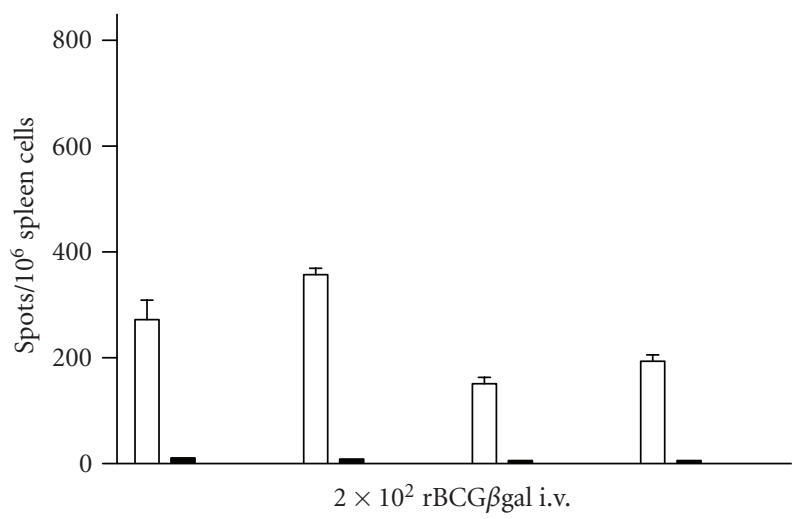

(c)

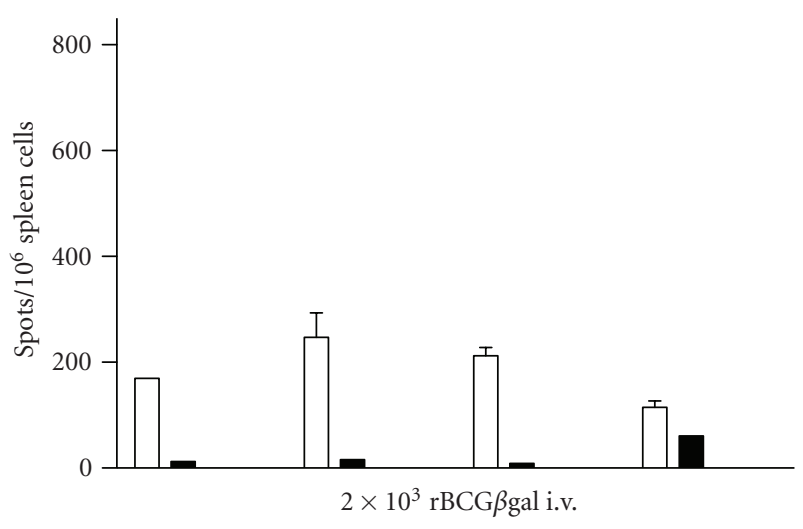

(b)

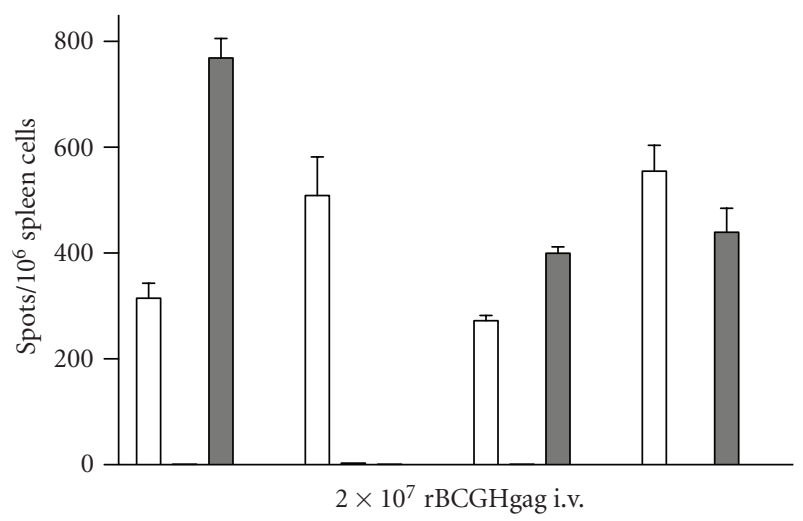

(d)

FIGURE 1: Immune response of four individual mice per group after i.v. immunization with different doses of recombinant BCG (rBCG $\beta$ gal) or control rBCG expressing HIV gag protein (rBCGHgag). Immunization dose is indicated below each graph. Mice were immunized as indicated under each graph. After 9 weeks, the mice were killed and the number of $\beta$-gal- and BCG-specific IFN $\gamma$ - and IL-4-producing cells in their spleens was determined by ELISPOT assay. The response in mice given a high dose of BCG transformed with a control vector containing the HIV gag sequence is also shown. Open bars-IFN $\gamma$ response to BCG; Black bars-IFN $\gamma$ response to $\beta$-gal; Dark gray barsIL-4 response to BCG; Light gray bars-IL-4 response to $\beta$-gal. All values were corrected for background by subtracting the number of spots observed in wells without antigen. Results are representative of two independent experiments.

electroporation-competent BCG. Transformation of competent BCG was performed by electroporation using the Gene Pulser II (BioRad). Electroporation-competent BCG was removed from the freezer and immediately placed on ice for several minutes to allow samples to thaw. One microgram of plasmid DNA was added to the tube, and the contents were mixed and placed on ice for ten minutes. The mixture was transferred to a chilled, $2 \mathrm{~mm}$ gap electroporation cuvette (VWR scientific, Toronto, ON). The bacteria were subjected to a pulse of $3.5 \mathrm{kV}, 25 \mu \mathrm{F}$, at a resistance of $1000 \Omega$ [12]. The cuvette was incubated on ice for ten minutes before the contents were transferred to a separate tube and diluted with $3 \mathrm{ml}$ of Middlebrook 7H9 media (Difco, Detroit, MI) with $0.5 \%$ Tween 80 and OADC supplement (Difco). The mixture was incubated at $37^{\circ} \mathrm{C}$ for $3 \mathrm{~h}$ to allow for antibiotic expression. Cells were harvested by centrifugation at $3000 \mathrm{~g}$ for ten minutes and plated on Middlebrook 7H10 agar plates with OADC and $10 \mu \mathrm{g} / \mathrm{ml}$ of kanamycin [12]. The plates were sealed with parafilm and incubated at $37^{\circ} \mathrm{C}$ for $21-28$ days to allow colonies to grow.

\section{Results}

Figures 1 and 2 show the number of BCG- and $\beta$-galspecific IFN $\gamma$ - and IL-4-producing cells in the spleens of mice nine weeks after immunization with different doses of rBCG (either rBCG $\beta$ gal or rBCGHgag). Immunization with both low and high doses of rBCG $\beta$ gal induced not only BCGspecific but also $\beta$-gal-specific cytokine-producing cells, indicating that the $\beta$-gal is expressed in vivo at a level sufficient to induce an immune response. The immune response to different doses of rBCG given by i.v. (Figure 1) and i.d. (Figure 2) immunization is qualitatively different. Regardless of the route, high doses of rBCG $\beta$ gal induce a mixed IFN $\gamma$ and IL-4 response while lower doses induce a predominantly IFN $\gamma$ response to both BCG and the expressed $\beta$-gal antigen. Although the numbers of $\beta$-galspecific spot forming cells are low in rBCG $\beta$ gal immunized mice, they represent a significant portion of the response to the $\mathrm{rBCG} \beta$ gal, particularly in mice immunized by the i.d. route. Mice immunized with BCG transformed with a control vector BCGHgag, not expressing $\beta$-gal (Figure 1(d)), 


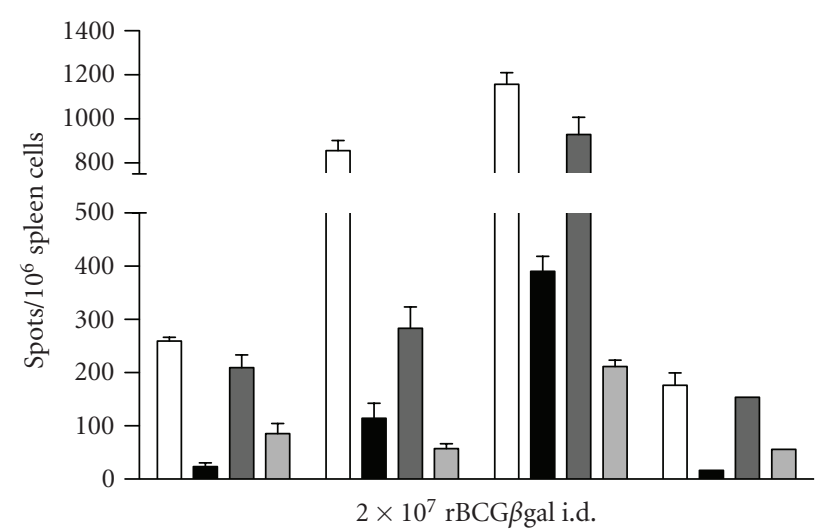

(a)

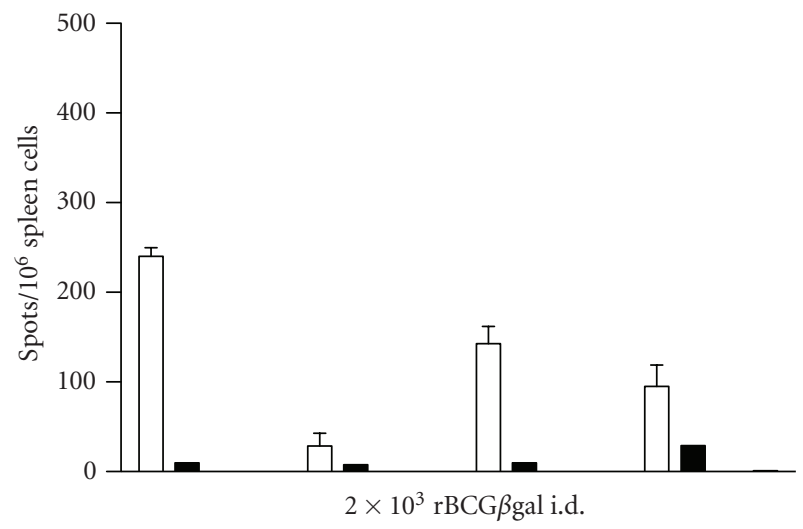

(c)

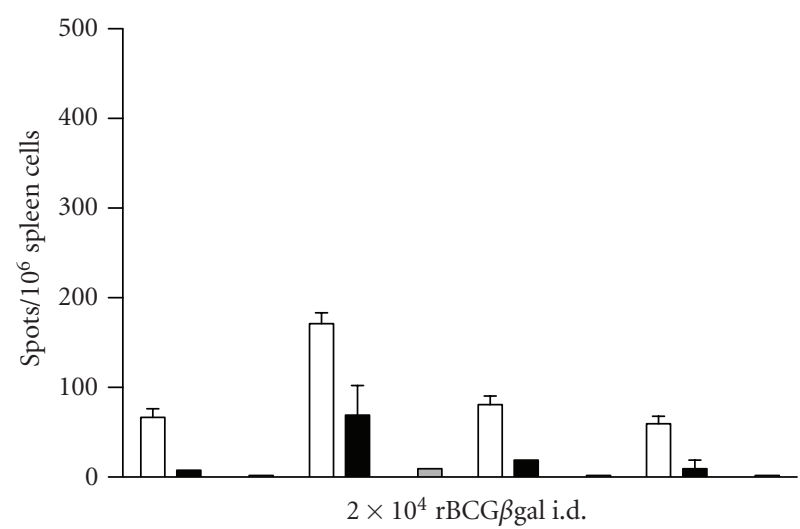

(b)

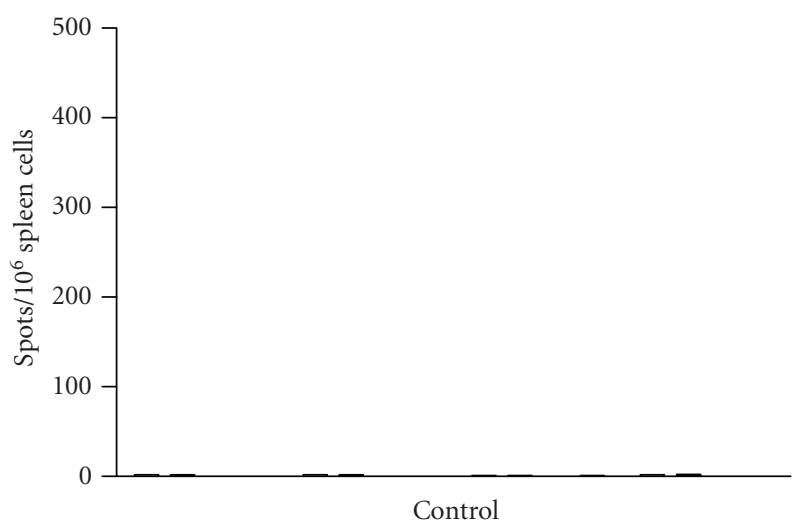

(d)

FIGURE 2: Immune response of four individual mice per group after i.d. immunization with different doses of recombinant BCG $\beta$ gal. Mice were immunized as indicated under each graph, with an unimmunized control shown. After 9 weeks, the mice were killed and the number of $\beta$-gal- and BCG-specific IFN $\gamma$ - and IL-4-producing cells in their spleens was determined by ELISPOT assay. Open bars-IFN $\gamma$ response to BCG; Black bars-IFN $\gamma$ response to $\beta$-gal; Dark gray bars-IL-4 response to BCG; Light gray bars-IL-4 response to $\beta$-gal. All values were corrected for background by subtracting the number of spots observed in wells without antigen. Results are representative of two independent experiments.

show no response to $\beta$-gal while cells from unimmunized control mice (shown in Figure 2(d)) do not respond to either the BCG-lysate antigen or to the $\beta$-gal antigen. Only mice immunized with the high doses of rBCG $\beta$ gal had significant antibody titers to $\beta$-gal (data not shown), indicating that the antibody response is indeed attributable to immunization with rBCG $\beta$ gal, and that only higher doses induce antibody production. Similarly, high doses of rBCG were required for induction of BCG-specific antibody production, demonstrating that the humoral response to $\beta$ gal follows that induced for the BCG.

We wished to determine if low-dose immunization with rBCG $\beta$ gal could establish immune deviation such that the IFN $\gamma$-dominant response initially induced by the low-dose vaccination would be maintained upon subsequent challenge with a dose of rBCG $\beta$ gal that induces a mixed IFN $\gamma / \mathrm{IL}-4$ response in naïve mice. Mice immunized with different doses of rBCG by the i.d. route were challenged four months later with a high dose of rBCG $\beta$ gal, also given intradermally. The immune response was assayed at 16 weeks postchallenge. Mice that were immunized with low doses by the i.d. route
(Figure 3) maintained a dominant IFN $\gamma$ response to BCG antigen and to $\beta$-gal after the challenge, which was distinct from the mixed IFN $\gamma / \mathrm{IL}-4$ response of control mice that received only the challenge dose, demonstrating immune deviation toward a cell-mediated response. The ratio of IFN $\gamma$ - to IL-4-producing cells (means within each group) are depicted in Figure 4. Even after challenge with the high dose of $\mathrm{rBCG} \beta$ gal, the mice that were immunized previously with a low dose of rBCG have a tenfold higher IFN $\gamma$ response compared to control mice or those mice given only the high challenge dose. These results demonstrate that, once established, the dominant IFN $\gamma$ response induced by low-dose vaccination is not easily deviated to an IL4 response upon challenge. The anti- $\beta$-gal serum antibody titers from the mice of Figure 3 are shown in Figure 5. Note the prominence of IgG2a antibody in the serum of mice immunized with a low dose of rBCG before challenge, compared to the control mice given only the high dose. This is consistent with the prominence of IFN $\gamma$-producing cells in mice pretreated with low doses of rBCG, as class switching to IgG2a is associated with IFN $\gamma$ production in mice [3]. 


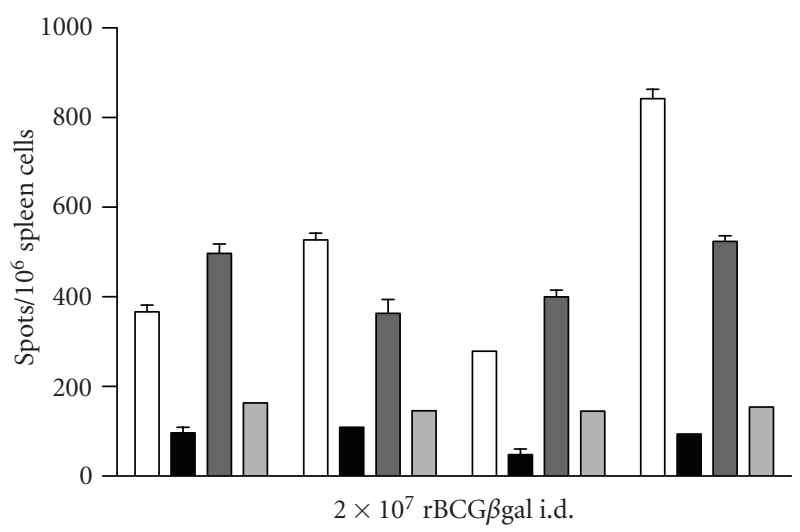

(a)

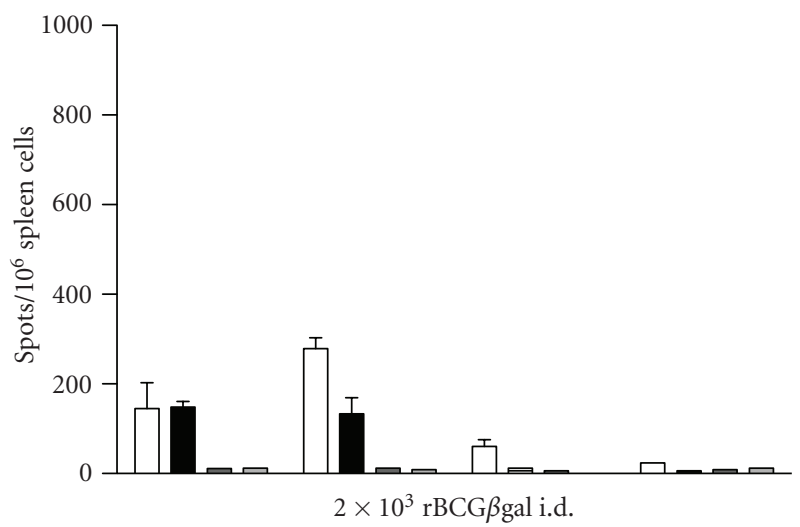

(c)

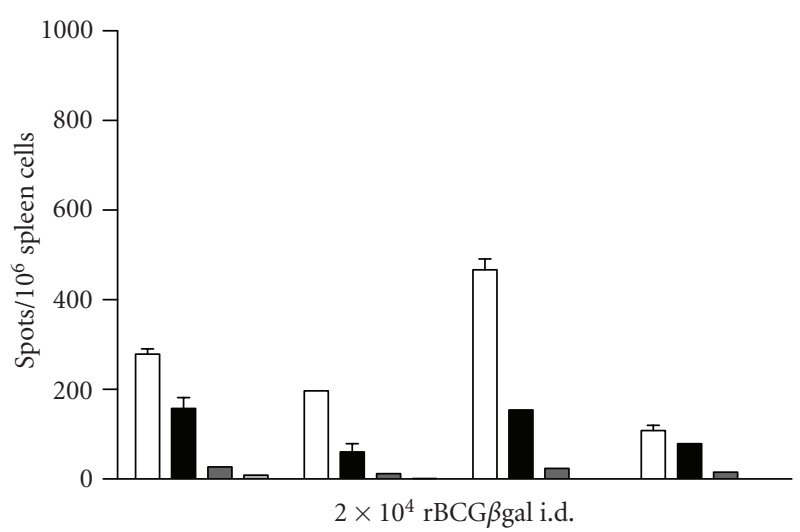

(b)

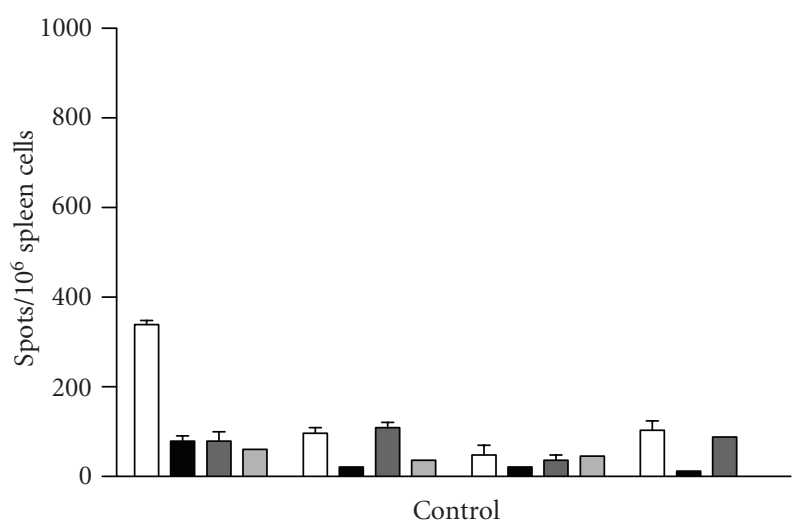

(d)

FIGURE 3: Immune response of individual mice vaccinated with different doses of rBCG $\beta$ gal after subsequent i.d. challenge with a high dose of rBCG $\beta$ gal. Each group of mice was initially given an i.d. vaccination dose of rBCG $\beta$ gal as indicated below each graph with a control group receiving challenge dose only shown. After 4 months, the mice were challenged with approximately $2 \times 10^{7} \mathrm{rBCG} \beta$ gal cfu given i.d. After an additional 16 weeks, the mice were killed and the number of $\beta$-gal- and BCG-specific IFN $\gamma$ - and IL-4-producing cells in their spleens was determined by ELISPOT assay. Open bars-IFN $\gamma$ response to BCG; Black bars-IFN $\gamma$ response to $\beta$-gal; Dark gray bars-IL-4 response to BCG; Light gray-IL-4 response to $\beta$-gal. All values were corrected for background by subtracting the number of spots observed in wells without antigen. Results are representative of two independent experiments.

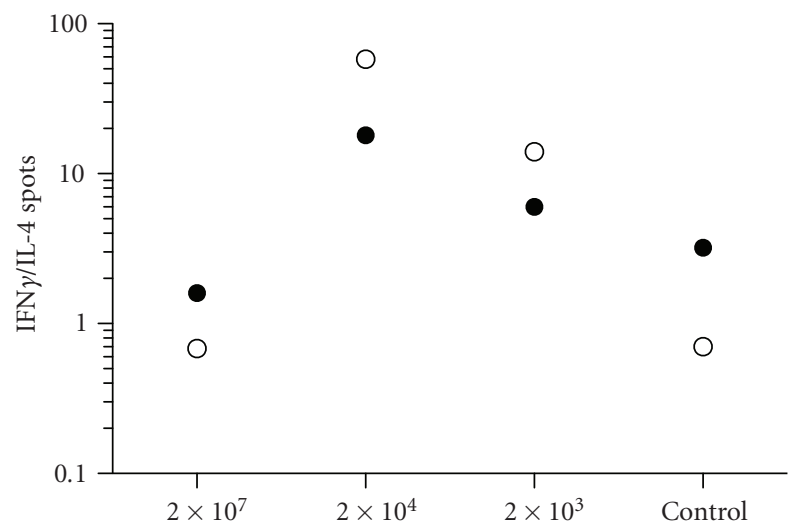

FIgURE 4: Ratio of IFN $\gamma$ to IL-4 spots in the mice depicted in Figure 3. Ratios were calculated as the mean number of antigenspecific IFN $\gamma$ spots/the mean number of IL-4 spots within each group of mice. Open circles and filled circles represent BCG- and $\beta$ gal-specific IFN $\gamma$ : IL-4 ratios, respectively.
We compared the immune response to $\beta$-gal in mice immunized with rBCG $\beta$ gal and in mice immunized with purified $\beta$-gal using other methods that might be acceptable for immunization of humans. Figure 6 shows the immune response in mice immunized with purified $\beta$-gal given in alum as an adjuvant. In both cases, immunization was by the i.p. route. Soluble $\beta$-gal did not induce a significant immune response in any of the mice immunized with this preparation (data not shown). $\beta$-gal in alum, however, induced predominantly IL-4-producing cells in mice, at even the low dose used. This is not unexpected as alum is particularly effective at inducing Th2 responses [13]. However, these results exemplify potential problems in achieving a desirable Th1 response in humans with current vaccination procedures.

\section{Discussion}

Previous studies have shown that the Th1/Th2 phenotype of the immune response to BCG, generated in very young and 


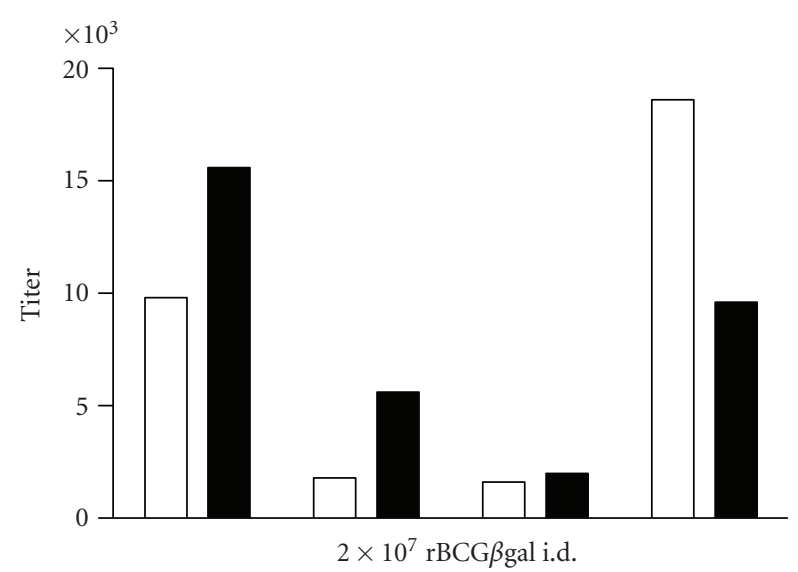

(a)

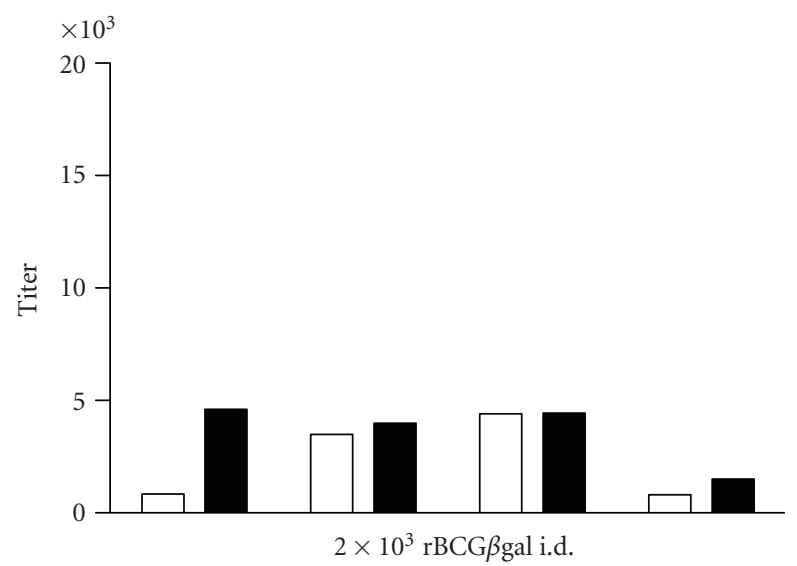

(c)

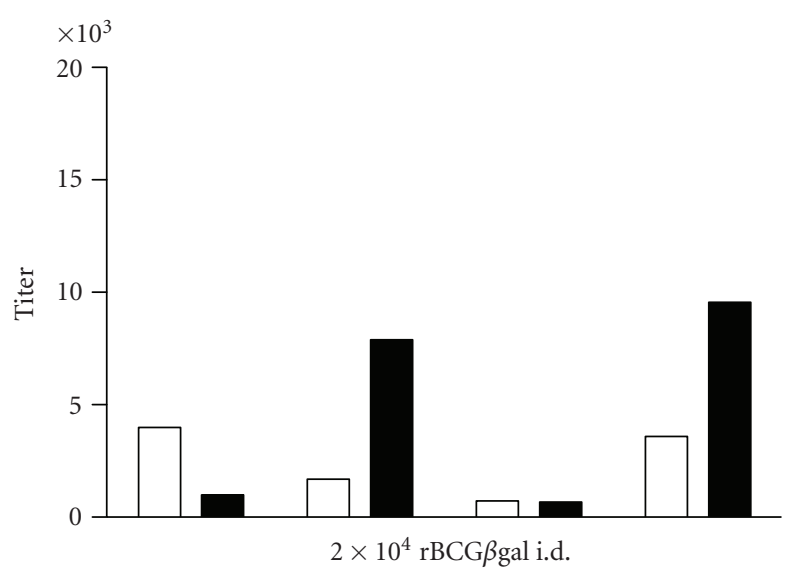

(b)

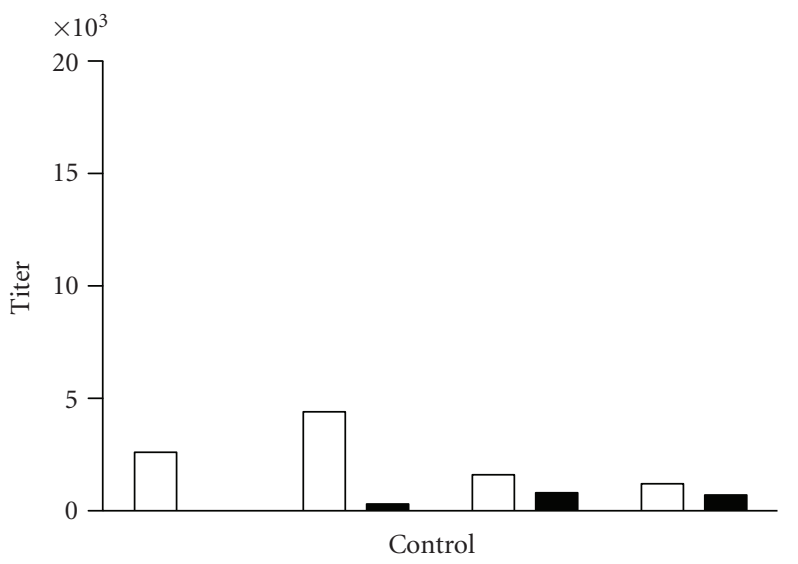

(d)

FIGURE 5: Serum antibody responses of mice to $\beta$-gal after immunization with different doses of rBCG by the i.d. route, and subsequent challenge with a high dose of rBCG by the same route. Mice were immunized initially with doses indicated below each graph with agematched control mice receiving only challenge dose shown. After 16 weeks, all groups were challenged with $2 \times 10^{7} \mathrm{rBCG}$ given i.d. Serum was collected at the time the mice were killed, 16 weeks after the challenge. Open bars show IgG1 titers while black bars depict IgG2a titers.

in adult mice, depends upon the dose of BCG administered, as assessed following infection by several different routes [10]. Infection with relatively low numbers of BCG generates a predominant Th1 response and with higher numbers a mixed Th1/Th2 response. Here we show that the Th1/Th2 phenotypes of the immune responses to BCG and to $\beta$-gal similarly depend upon the number of rBCG employed for infection and that the Th1/Th2 phenotypes of the responses to BCG and to $\beta$-gal appear to be coherent. Our studies show that rBCG can be used to preferentially generate a Th1 response to the encoded antigen by infecting with relatively low doses of rBCG. We now consider the potential use of this strategy in vaccination against HIV-1 and influenza viruses.

In looking at current views, it appears that there are two main, contradictory, positions as to how effective vaccination against HIV-1 might be achieved. Most suggest the combination of cell-mediated immunity, in the form of CTL and neutralizing antibody, will provide optimal protection. Thus, vaccination should ensure such a response is mounted upon natural exposure to the virus. It is well known that neutralizing antibody can be generated, but that HIV-1 has a means of generating viral variants very quickly. As such, neutralizing antibody generated against the original variant encountered will be effective against this particular variant, but usually is ineffective against newly arising variants that become dominant through the course of the infection.

The contrasting view we favor is that vaccination, in order to be effective, must guarantee a predominant Th1 response, associated with potent CTL generation, upon HIV-1 infection. We think three lines of reasoning support this view. Firstly, there are highly exposed and infected individuals who produce predominant and stable Th1, CTL responses, but who are symptom-free. A prime illustration of such a group is a cohort of female sex workers in Nairobi, whose immune state has been extensively characterized [14, $15]$. What is additionally remarkable about this group of individuals is that they do not seroconvert and remain healthy over many years despite numerous exposures to major viral variants or clades of HIV-1 due to the nature of their work. Secondly, all individuals infected with HIV1 are healthy during the period when their immune system 


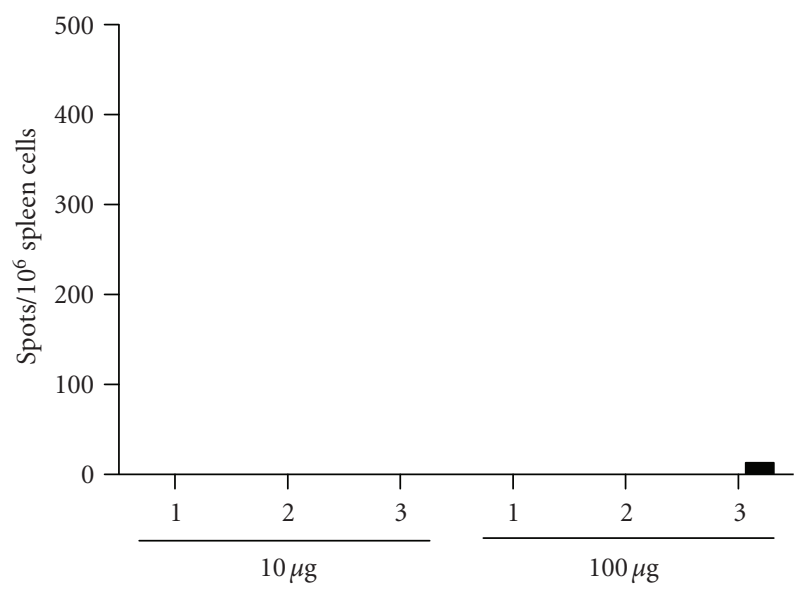

(a)

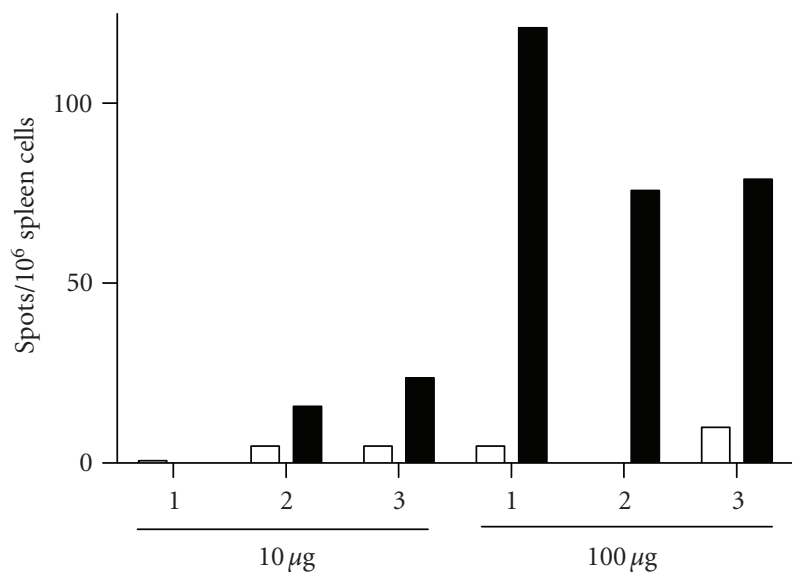

(b)

FIgURE 6: Number of IFN $\gamma$ - and IL-4-producing cells in mice immunized i.p. with soluble $\beta$-gal (A) and $\beta$-gal adsorbed to alum as an adjuvant (B). Soluble $\beta$-gal was administered twice five days apart, while $\beta$-gal/alum was administered only once. Dose of $\beta$-gal administered in each experiment is indicated $(10 \mu \mathrm{g}$ or $100 \mu \mathrm{g})$. Mice were killed 11 days after the initial injection, and antigen-specific cytokine-producing spleen cells were enumerated by ELISPOT assay. Open bars represent IFN $\gamma$ spots and black bars represent IL-4 spots. Results are representative of three separate experiments.

mounts a predominant Th1, CTL response, before they seroconvert and enter the progressive stages of disease [16]. Both observations strongly support the idea that a predominant Th1, CTL response can contain HIV-1. Why then not aim for having the best of both worlds, both CTL and neutralizing antibody, as most suggest? It is an assumption, and one that we feel is highly questionable, that this is possible. Distinct $\mathrm{CD}^{+}{ }^{+} \mathrm{T}$ cell subsets tend to inhibit each others' generation and often each others' effector activities [1]. It may not be possible to have the best of both worlds, involving a mixed Th1/Th2 response, with optimal production of CTL immunity and neutralizing antibody. We think it best to conclude, in accordance with natural situations in which the virus is contained, and infected individuals remain symptom-free, that a predominant Th1, CTL response is optimally protective. We employ this proposition as a starting point for considering how effective vaccination might be achieved.

In the case of influenza virus, that causes an acute infection, it seems antibody can be highly protective. Antibody is usually not produced in sufficient amounts upon infection of naïve individuals to prevent pathological symptoms, which is why immunization against the virus causing the current epidemic is effective in preventing illness. Individuals who have been infected during the last epidemic, but not immunized against the virus causing the current epidemic, are highly primed to produce antibody. The antibody produced is highly effective in neutralizing the influenza strain causing the previous epidemic, but not the virus causing the current epidemic. This makes eminent sense from an epidemiological point of view. It is just because the current influenza strain can stimulate the production of this antibody, ineffective in its own neutralization, that the virus can multiply unimpeded in the individual, primed by a previous infection, with the consequential spread of the virus through the population. An appreciation of these facts underlies the formulation of current policy. The aim is to achieve immunization against the strain causing the current epidemic as soon as it has been identified. This policy is sound, but its realization is extremely expensive and poses considerable logistical problems, clearly illustrated throughout the most recent influenza $\mathrm{H} 1 \mathrm{~N} 1$ pandemic.

The CTL response against influenza involves, in large measure, a response against peptides derived from viral proteins that do not vary greatly between strains, such as peptides derived from the nuclear protein, NP [17]. The possibility might therefore be considered that vaccination resulting in a sustained and effective CTL response upon infection by diverse variants might be effective against all variants. An intriguing report supports this possibility. DNA immunization of mice against NP results in a predominant Th1, CTL response on challenge with different viral variants and resistance [18]. Thus generating stable Th1 imprints against influenza might result in protection against diverse viral variants.

The observations reported here and our considerations on how vaccines against diverse clades of HIV-1 and strains of influenza might be achieved by causing Th1 imprints lead us to suggest that attempts at realizing a low-dose rBCG vaccination strategy is worthy of further exploration. We showed in this paper that infection with low numbers of rBCG generated not only predominant Th1 responses but also Th1 imprints as assessed by the predominant Th1 responses observed upon a subsequent challenge with a high number of $\mathrm{rBCG}$ that generate mixed Th1/Th2 responses in naïve animals. We consider the next important step in assessing the plausibility of this strategy will be to examine whether $\mathrm{rBCG}$ can provide protection against a pathogenic infection. There are mouse models of influenza infection. We hope to examine, employing established systems, whether infection of mice with low numbers of rBCG vectors, encoding conserved proteins of influenza virus, can generate Th1 imprints, as assessed by infection with a normally 
pathogenic challenge of influenza virus, and whether such imprints provide protection against diverse viral variants.

\section{Acknowledgments}

The authors thank Dr. Mark Hansen for providing the mycobacterial plasmids and D. Yurkowski for valuable technical assistance. This work was supported by a CIHR grant held by Peter Bretscher.

\section{References}

[1] P. A. Bretscher, N. Ismail, J. N. Menon, C. A. Power, J. Uzonna, and G. Wei, "Vaccination against and treatment of tuberculosis, the leishmaniases and AIDS: perspectives from basic immunology and immunity to chronic intracellular infections," Cellular and Molecular Life Sciences, vol. 58, no. 12-13, pp. 1879-1896, 2001.

[2] D. H. Hamilton and P. A. Bretscher, "Different immune correlates associated with tumor progression and regression: implications for prevention and treatment of cancer," Cancer Immunology, Immunotherapy, vol. 57, no. 8, pp. 1125-1136, 2008.

[3] P. A. Bretscher, G. Wei, J. N. Menon, and H. BielefeldtOhmann, "Establishment of stable, cell-mediated immunity that makes "susceptible" mice resistant to Leishmania major," Science, vol. 257, no. 5069, pp. 539-542, 1992.

[4] N. Ohara and T. Yamada, "Recombinant BCG vaccines," Vaccine, vol. 19, no. 30, pp. 4089-4098, 2001.

[5] C. R. Parish, "The relationship between humoral and cellmediated immunity," Transplantation Reviews, vol. 13, pp. 3566, 1972.

[6] B. M. Buddle, G. W. De Lisle, A. Pfeffer, and F. E. Aldwell, "Immunological responses and protection against Mycobacterium bovis in calves vaccinated with a low dose of BCG," Vaccine, vol. 13, no. 12, pp. 1123-1130, 1995.

[7] T. G. Kiros, et al., "Immunization of newborn and adult mice with low numbers of BCG leads to Th1 responses, Th1 imprints and enhanced protection upon BCG challenge," Immunotherapy, vol. 2, no. 1, pp. 25-35, 2010.

[8] A. Lotte, O. Wasz-Hockert, N. Poisson, et al., "Second IUATLD study on complications induced by intradermal BCG-vaccination," Bulletin of the International Union Against Tuberculosis and Lung Disease, vol. 63, no. 2, pp. 47-59, 1988.

[9] C. A. Power, C. L. Grand, N. Ismail, N. C. Peters, D. P. Yurkowski, and P. A. Bretscher, "A valid ELISPOT assay for enumeration of ex vivo, antigen-specific, IFN $\gamma$-producing $\mathrm{T}$ cells," Journal of Immunological Methods, vol. 227, no. 1-2, pp. 99-107, 1999.

[10] C. A. Power, G. Wei, and P. A. Bretscher, "Mycobacterial dose defines the Th1/Th2 nature of the immune response independently of whether immunization is administered by the intravenous, subcutaneous, or intradermal route," Infection and Immunity, vol. 66, no. 12, pp. 5743-5750, 1998.

[11] C. K. Stover, V. F. De La Cruz, T. R. Fuerst, et al., "New use of BCT for recombinant vaccines," Nature, vol. 351, no. 6326, pp. 456-460, 1991.

[12] T. Parish and N. G. Stoker, "Electroporation of mycobacteria," in Molecular Biology: Electroporation of microorganisms, J. A. Nickoloff, Ed., pp. 237-252, Human Press, Totowa, NJ, USA, 1995.
[13] J. L. Grun and P. H. Maurer, "Different T helper cell subsets elicited in mice utilizing two different adjuvant vehicles: the role of endogenous interleukin 1 in proliferative responses," Cellular Immunology, vol. 121, no. 1, pp. 134-145, 1989.

[14] K. R. Fowke, N. J. D. Nagelkerke, J. Kimani, et al., "Resistance to HIV-1 infection among persistently seronegative prostitutes in Nairobi, Kenya," Lancet, vol. 348, no. 9038, pp. 1347-1351, 1996.

[15] K. R. Fowke, R. Kaul, K. L. Rosenthal, et al., "HIV-1specific cellular immune responses among HIV-1-resistant sex workers," Immunology and Cell Biology, vol. 78, no. 6, pp. 586$595,2000$.

[16] J. Cohen, "AIDS research shifts to immunity," Science, vol. 257, no. 5067, pp. 152-154, 1992.

[17] J. B. Ulmer, J. J. Donnelly, S. E. Parker, et al., "Heterologous protection against influenza by injection of DNA encoding a viral protein," Science, vol. 259, no. 5102, pp. 1745-1749, 1993.

[18] S. L. Epstein, W.-P. Kong, J. A. Misplon, et al., "Protection against multiple influenza A subtypes by vaccination with highly conserved nucleoprotein," Vaccine, vol. 23, no. 46-47, pp. 5404-5410, 2005. 


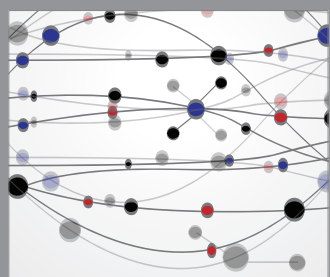

The Scientific World Journal
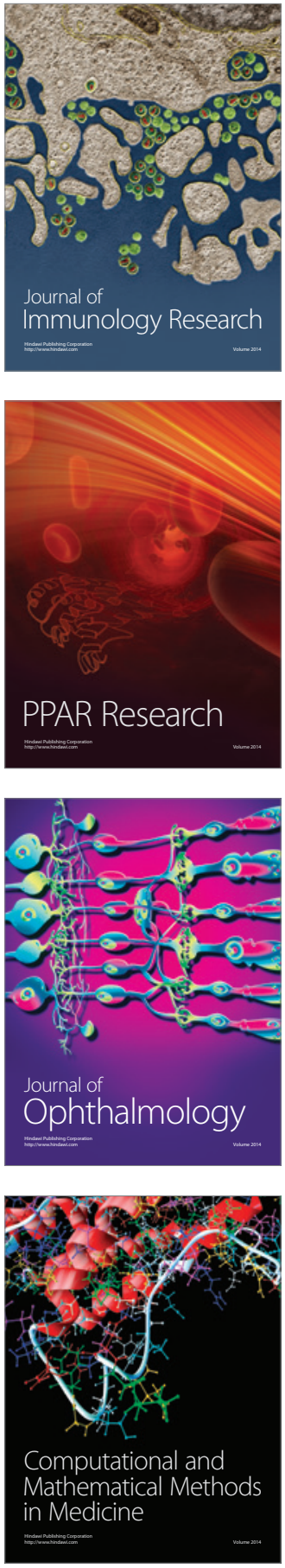

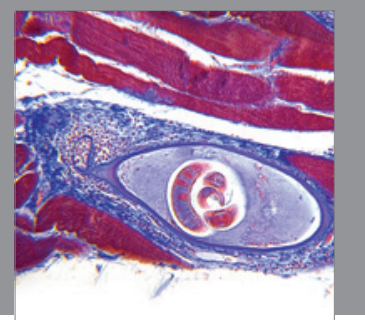

Gastroenterology

Research and Practice
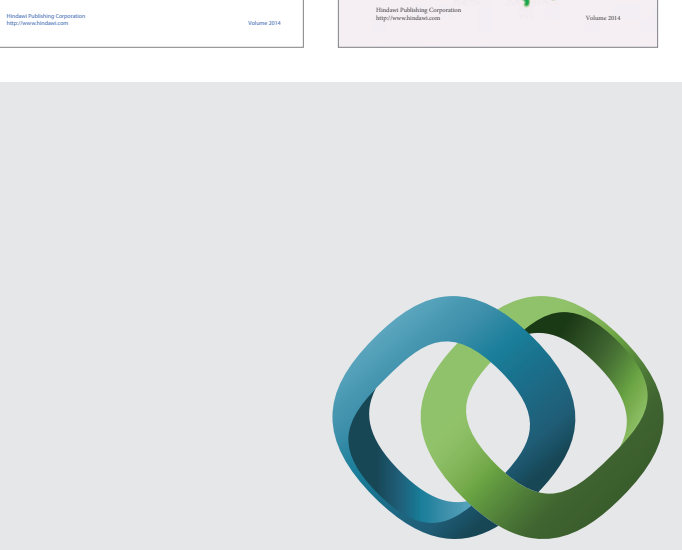

\section{Hindawi}

Submit your manuscripts at

http://www.hindawi.com
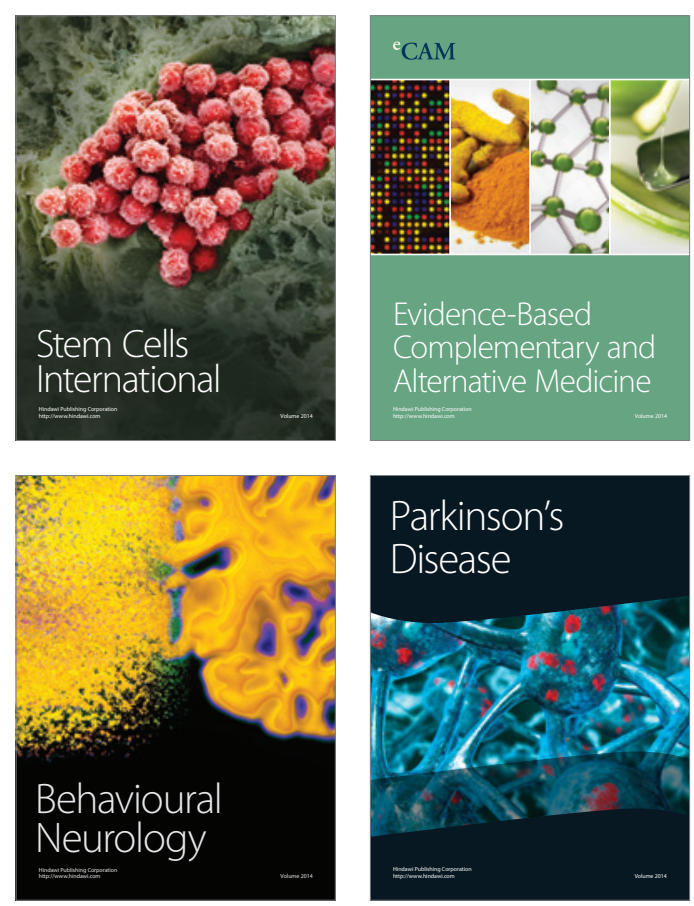

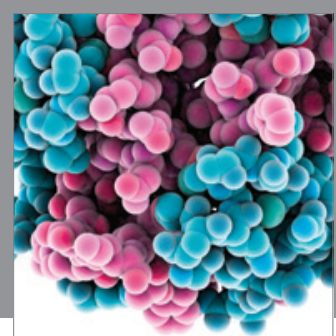

Journal of
Diabetes Research

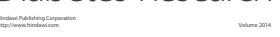

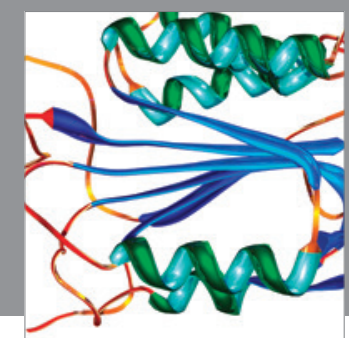

Disease Markers
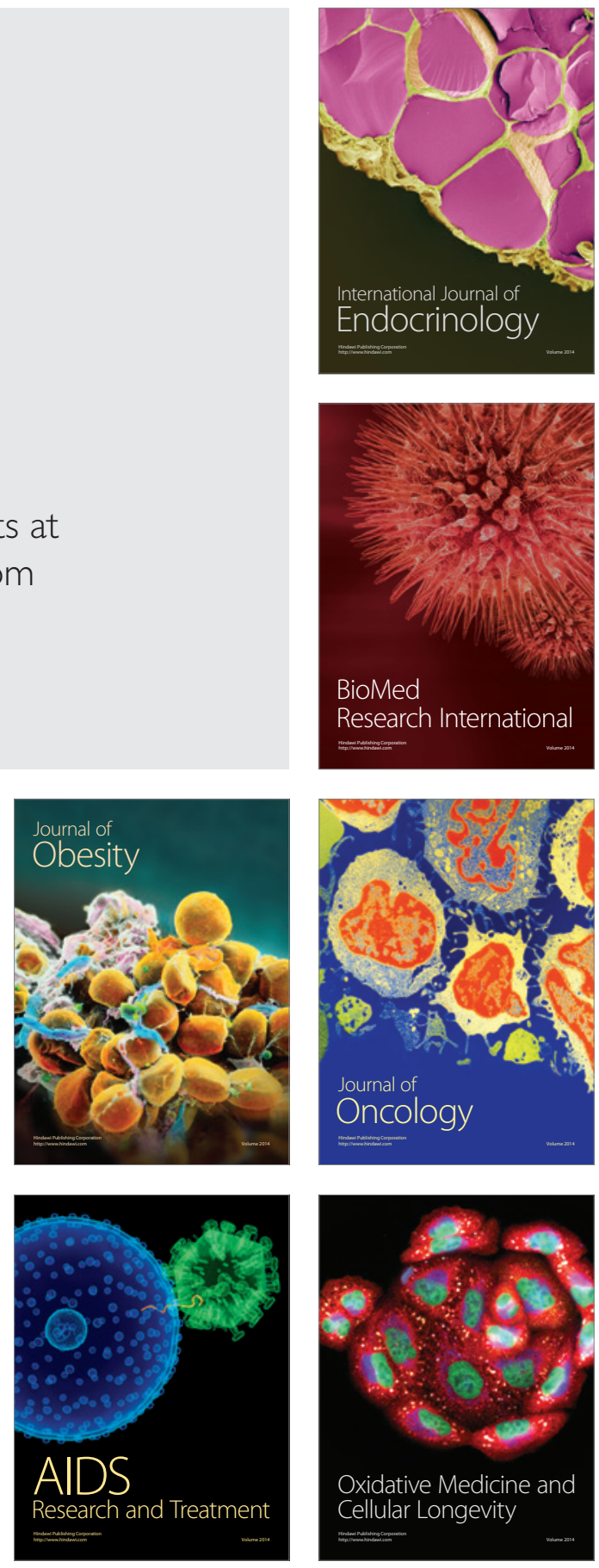